FERMILAB-TM-2453-DI

October 2009

\title{
Accelerator/Experiment Operations - FY 2009
}

\author{
M. Andrews, J. A. Appel, S. Brice, M. Casarsa, R. Coleman, D. Denisov, G. Ginther, \\ S. Gruenendahl, S. Holmes, W. Kissel, W. M. Lee, R. Mau, C. Moore, R. Plunkett, \\ E. Ramberg, P. Schlabach, S. Soldner-Rembold, R. Van de Water, C. White
}

Edited by J. A. Appel and J. Coleman

This Technical Memorandum (TM) summarizes the Fermilab accelerator and accelerator experiment operations for FY 2009. It is one of a series of annual publications intended to gather information in one place. In this case, the information concerns the FY 2009 Run II at the Tevatron Collider, MINOS using the Main Injector Neutrino Beam (NuMI), the MiniBooNE experiment running in the Booster Neutrino Beam (BNB), and the Meson Test Beam (MTest) activities in the $120 \mathrm{GeV}$ external Switchyard beam (SY120).

Each section was prepared by the relevant authors, and was somewhat edited for inclusion in this summary.

Accelerator Operations (M. Andrews, J. A. Appel, S. Holmes, R. Mau)

\section{$\underline{\text { Tevatron Collider }}$}

The Fermilab accelerators entered the fiscal year continuing the very long running period since October 28, 2007, the end of the previous major shutdown. The next long shutdown began June 15, 2009. Thus, the running lasted nearly 20 months. The 2009 fiscal year ended with the accelerators coming back from the latter shutdown, on a path to the stable running with the excellent luminosity that characterized most of the fiscal year. The long shutdown itself was executed as scheduled, in spite of needing to warm up nine Tevatron cryogenic houses for repair (though only six were on the schedule at the start of the shutdown). See more details on the shutdown activity at the end of this section of the report (Table 2).

For FY 2009 (defined as 0000 on October 6, 2008 to 2400 on October 4, 2009), integrated luminosity (average of CDF and DZero) totaled $1905.7 \mathrm{pb}^{-1}, 108 \%$ of the increased design goal developed after the start of the fiscal year, and 106\% of that delivered in FY 2008 despite nine fewer weeks of running. Over the 39 weeks of scheduled Collider operations, 6221 store hours were delivered to CDF and DZero, $106 \%$ of the design goal. Total on-line average integrated luminosity for Run II stood at $6.90 \mathrm{fb}^{-1}$ as of 10/05/09.

\section{Commentary on FY 2009 Collider Performance}

The Run II Improvement Plan was completed in 2007. Thus, the current strategy is to maintain reliable operations at the current excellent level of performance. 
Highlights for the year include:

- Average luminosity delivered to CDF and D0 (10/06/08 through 10/05/09) significantly exceeded even the design curve: $1905.7 \mathrm{pb}^{-1}$ (see Figure 1)

- Reliability above design goal: average of 108 hours/week of luminosity

- Shutdown initiated as planned June 15, 2009; completed on schedule (11 weeks plus two weeks of Collider startup)

- Peak luminosity increased $11 \%\left(318 \rightarrow 353 \times 10^{30} \mathrm{~cm}^{-2} \mathrm{sec}^{-1}\right)$

- Peak weekly integrated luminosity up $28 \%\left(57 \rightarrow 73 \mathrm{pb}^{-1}\right)$

- Peak monthly integrated luminosity up $19 \%\left(221 \rightarrow 264 \mathrm{pb}^{-1}\right)$

- Peak antiproton stacking up $6 \%\left(27.0 \rightarrow 28.6 \times 10^{10} / \mathrm{hr}\right)$

- Weekly accumulated antiprotons up $25 \%\left(3000 \rightarrow 3770 \times 10^{10} /\right.$ week $)$

- 6369 hours scheduled uptime (10/04/08 through 10/06/09)

- Unscheduled downtime (1035 hours, including the three weeks after the long scheduled shutdown): $16.3 \%$

- Significant downtime contributors:

- Power outage

- Transformer failure

- CHL compressor failure

- All were managed well and limited associated downtime to $<1$ week

- 4195 Tevatron HEP-store hours (10/04/08 through 10/06/09)

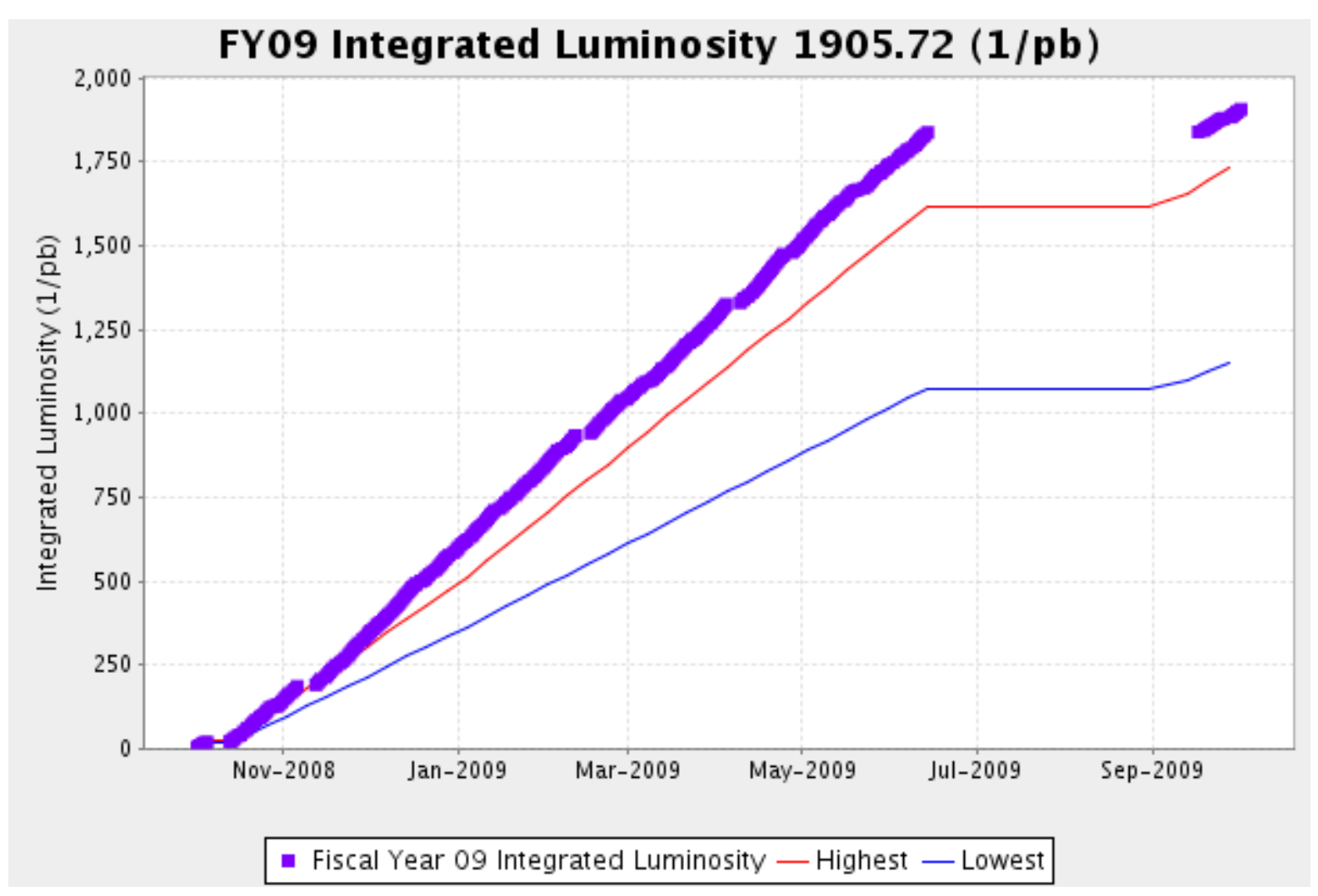

Figure 1. FY 2009 integrated luminosity, planned and delivered (average of CDF and DZero). 
In December, the Collider operating mode was changed. New stores are now initiated when there are enough antiprotons available to increase the delivered luminosity over the following two hours beyond what would be obtained by continuing to run the then-current store during those two hours. This has led to shorter stores and an increased rate of integrating luminosity.

The Accelerator Division continued to reduce the time for shot setup by making improvements to the halo-scraping procedure (from Store 6715 onward), and by going to twobatch injection of protons into the Tevatron (from Store 6723 onward). The first step of scraping was shortened by a few minutes by moving collimators closer to the beam at an initial higher speed, before slowing down for fine tuning based on beam-loss feedback. The initial collimator moves are based on beam position measurements. Roughly 11.5 minutes were required to remove beam halo before speeding up; roughly 8.6 minutes afterward. Shortly after that improvement, proton injection was changed to use a two-batch mode, leading to proton injection times of $\sim 7$ minutes, compared to the previous $\sim 18-20$ minutes.

In March, a new capability, "short mining" of antiprotons from the Recycler Ring, was introduced. It is useful in the event that there are more antiprotons available than can be used for the next store. This allows higher antiproton stacking efficiency by keeping the stack of antiprotons in the Accumulator Ring small, and takes advantage of the extra capacity of the Recycler Ring to store antiprotons. This capability was first used for Store 6944 with 380 of $469 \times 10^{10}$ antiprotons mined for the store.

In April, the cooling of antiprotons in the Recycler Ring was improved by changes in the 2-4 GHz system. Also, new vertical and horizontal dampers were installed in the Recycler Ring. These improvements allow more frequent transfers to the Recycler Ring without sacrificing efficiency. Making transfers from smaller stacks in the Accumulator Ring improves the net antiproton accumulation rate. In tests, the transfers were started when the stacks reached levels at 35,30 , and $25 \times 10^{10}$ antiprotons. Starting transfers when the stacks were at $35 \times 10^{10}$ antiprotons in the Accumulator Ring, the antiproton accumulation rate was about $26.5 \times 10^{10}$ antiprotons/hour, while at $25 \times 10^{10}$ antiprotons, the rate climbed to $27.3 \times 10^{10}$ antiprotons/hour. After these tests, the operation was standardized with transfers from $25 \times 10^{10}$ antiprotons in the Accumulator Ring.

Reliability in FY 2009 was good. For the year, an average of 108 hours of luminosity per scheduled week of operations was reached - a 3\% increase from FY 2008. The antiproton stacking rate is the primary key to performance in the Tevatron. The design luminosity goal for 2009 was based on a stacking rate of $30 \times 10^{10}$ /hour. As noted above, a peak of $28.6 \times 10^{10} /$ hour was achieved. Significant modifications to the accelerator complex for the Collider program are complete. The future emphasis of Collider operations will be on reliability in order to maximize integrated luminosity in the Tevatron.

Antiproton availability remains the most important variable determining the luminosity of any particular store. Current experience is that luminosities in the range $2.7-3.2 \times 10^{32}$ are sustainable with a $25-26 \times 10^{10}$ /hour stacking rate as long as stores remain in the Tevatron for 15 18 hours. For the balance of Run II, the luminosity is expected to be roughly proportional to the stacking rate. 


\section{Status Relative to the FY 2009 Plan}

Table 1 shows the planned and actual performance for the year (defined as from the first Monday of the fiscal year through the first Sunday after the end of the fiscal year).

Table 1. 2009 Tevatron Collider planned and actual performance. Note that the Base and Design Profiles given here are the final ones, and are increased goals developed after the start of the fiscal year.

\begin{tabular}{|l|c|c|c|}
\hline & Base Profile & Design Profile & Actual* \\
\hline Median initial luminosity $\left(\mathrm{cm}^{-2} \mathrm{sec}^{-1}\right)$ & $1.9 \times 10^{32}$ & $3.1 \times 10^{32}$ & $3.1 \times 10^{32}$ \\
\hline Protons/bunch & $265 \times 10^{9}$ & $280 \times 10^{9}$ & $251 \times 10^{9}$ \\
\hline Pbars/bunch & $42 \times 10^{9}$ & $96 \times 10^{9}$ & $81.7 \times 10^{9}$ \\
\hline Accumulator-Recycler transfer efficiency & $91 \%$ & $92 \%$ & $95.5 \%$ \\
\hline Typical peak stacking rate (mA/hour) & 23 & 30 & 25 \\
\hline FY 2009 integrated luminosity (pb $\left.{ }^{-1}\right)$ & 1167 & 1762 & 1906 \\
\hline FY 2009 integrated store time (hours) & 3300 & 3960 & 4195 \\
\hline FY 2009 scheduled uptime (hours) & & & 6369 \\
\hline FY 2009 unscheduled downtime (hours) & & & 1035 \\
\hline $\begin{array}{l}\text { FY 2009 unscheduled downtime as percent of } \\
\text { scheduled uptime }\end{array}$ & & & $16.3 \%$ \\
\hline
\end{tabular}

* "Base" and "Design" labels correspond to "end-of-year" goals extended, where appropriate, to the standard reporting periods (Mondays through Sundays). "Actual" values in the first five rows correspond to simultaneous performance on the median store of the last month with four weeks before the long shutdown of FY 2009 (May). The goals for Base/Design integrated luminosity per week were $31 / 48 \mathrm{pb}^{-1}$, and store hours of $100 / 120$ hours/week, for fully scheduled weeks.

\section{FY 2009 Neutrino Operations}

The Proton Improvement Plan was fully implemented with the installation of the last complement of Booster correctors during the long FY 2009 accelerator shutdown. So far, however, the correctors are being operated without taking full advantage of the flexibility they have for sextupole corrections.

Typical beam on the NuMI target was approximately $260 \mathrm{~kW}$ during joint operation with antiproton production for the Collider program. This was achieved using 9 of the 11 Booster slipstacked batches in the Main Injector. The major downtime during the fiscal year was due to Horn 2 replacement. Nevertheless, the unscheduled downtime was somewhat smaller than anticipated.

The total of delivered protons to NuMI exceeded the base curve, and reached the DOE's Joule goal; $2.24 \times 10^{20}$ delivered protons on target $\left(10 / 04 / 08\right.$ through $10 / 06 / 09,2.18 \times 10^{20}$ during FY 2009, see Figure 2) compared to the goal of $2.2 \times 10^{20}$. The total of delivered protons was modestly higher than the $2.0 \times 10^{20}$ delivered protons in FY 2008, in spite of the shorter scheduled running time. There were long periods of very stable running for the NuMI beam. For the 7-day 
period of April 14-20, $8 \times 10^{18}$ protons were delivered; for the period between January 1 and May $31,0.29 \times 10^{20}$ protons were delivered per month; and between December 8 and May 31, the average beam power was $223 \mathrm{~kW}$. Since the beginning of NuMI operations in 2005, the total protons delivered now stands at $7.8 \times 10^{20}$.

Efforts continued on the mitigation of tritium production and releases from the NuMI facility. There were no measurable concentrations of tritium in any of the streams flowing off the Fermilab site boundaries in FY 2009. An Absorber Hall dehumidification system and a second evaporator were installed in the FY 2009 shutdown and are now operational. This system is expected to reduce future tritium levels by $40 \%$ in the ground water pumped from NuMI into the surface holding tank. Tritium levels in all surface ponds continue to be below detectable levels with the exception of Kidney Pond which is typically $2-5 \mathrm{pC} / \mathrm{ml}$ - slightly above detectable levels and well isolated from outflowing streams.

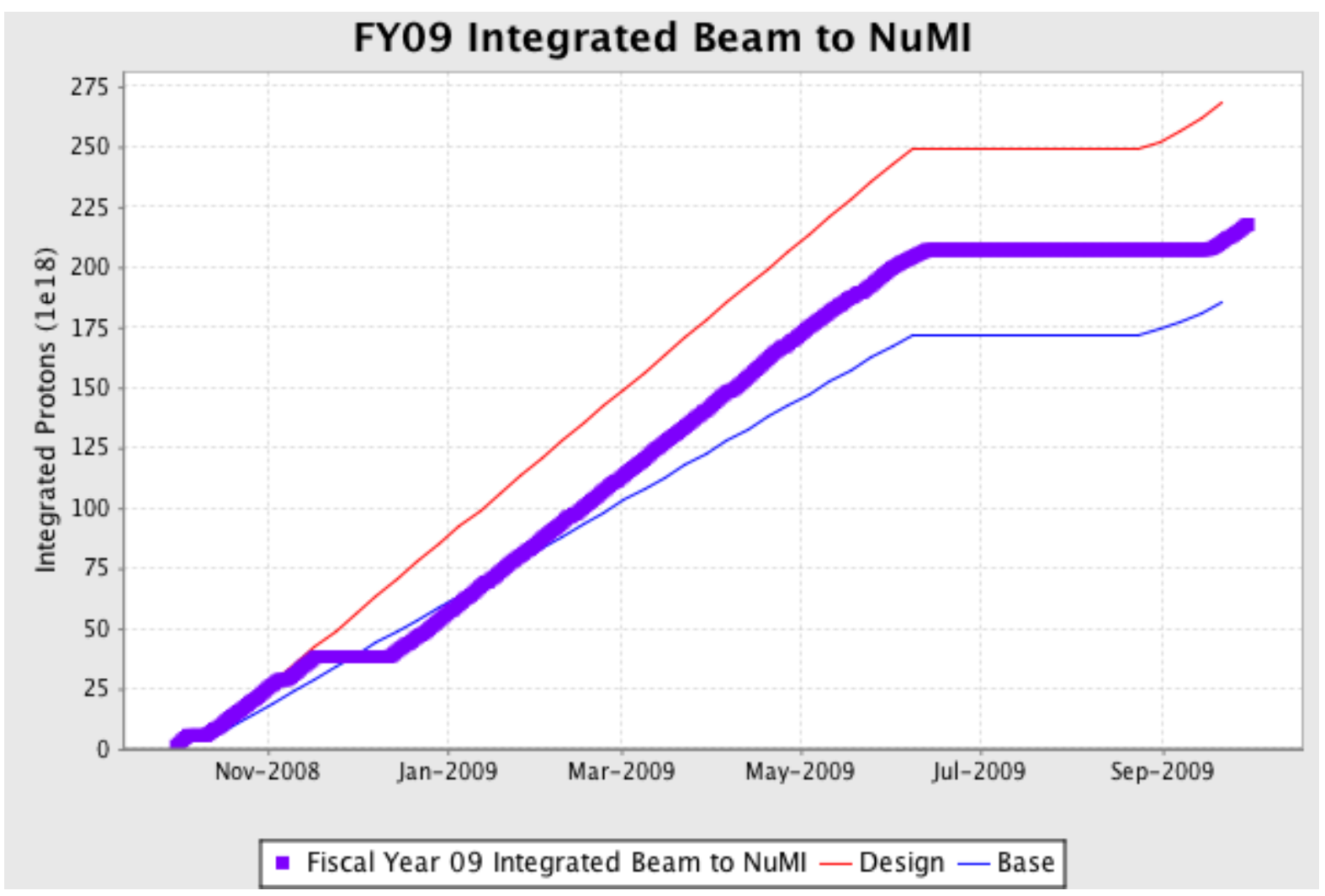

Figure 2. FY 2009 Integrated protons on target to NuMI.

The Booster Neutrino Beam operation for MiniBooNE benefited from interruption to NuMI operations. However, most of the $1.5 \times 10^{20}$ protons during the year (Figure 3) came by running in conjunction with NuMI. MiniBooNE finished the year essentially at their design level. MiniBooNE has accumulated data from a total of $13.5 \times 10^{20}$ protons delivered, and continued to run in antineutrino mode. 


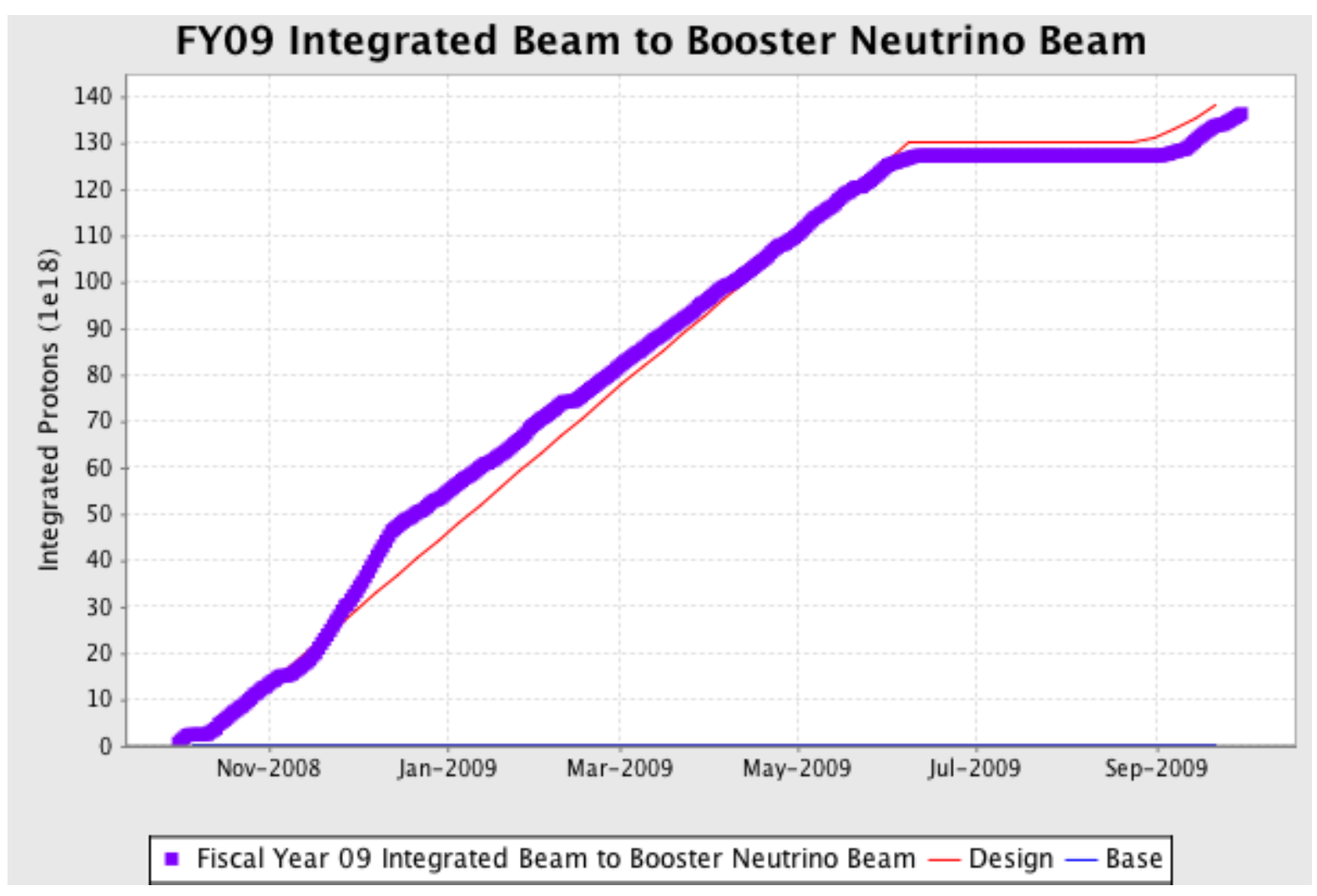

Figure 3. FY 2009 Integrated protons on target to MiniBooNE.

Table 2. Status of major shutdown activities.

\begin{tabular}{|l|l|l|}
\hline Machine/Activity & Goal & Status \\
\hline \hline Linac & & \\
\hline LLRF upgrade & $\begin{array}{l}\text { Improved flexibility and reliability. } \\
\text { A Proton Plan activity. }\end{array}$ & Complete \\
\hline Replace Linac drift tube & Improved reliability & Complete \\
\hline MTA 400 MeV beamline & $\begin{array}{l}\text { Installation of components to allow } \\
\text { delivery of 400 MeV beam to the } \\
\text { MuCool Test Area. }\end{array}$ & $\begin{array}{l}\text { All components installed. Final radiation } \\
\text { shielding assessment is currently under } \\
\text { way. }\end{array}$ \\
\hline Booster & & \\
\hline New Booster correctors & & $\begin{array}{l}\text { Loss reduction during acceleration. } \\
\text { A Proton Plan activity. }\end{array}$ \\
\hline $\begin{array}{l}\text { Upgrade of Booster Ring magnet power } \\
\text { supply system }\end{array}$ & Improved reliability & $\begin{array}{l}\text { Successful, with adjustments being made as } \\
\text { the intensity increased. }\end{array}$ \\
\hline Install Long 12 kicker & Improved reliability & $\begin{array}{l}\text { Installed new 13,800 volt switch and a new } \\
\text { transformer. }\end{array}$ \\
\hline $\begin{array}{l}\text { Antiproton Source } \\
\text { Replace leaking large quadrupole in the } \\
\text { transport line }\end{array}$ & Reliability risk reduction & Complete \\
\hline Install 2 momentum collimators & Allow increased antiproton yield \\
\hline $\begin{array}{l}\text { Manifold HV100 string: replace } \\
\text { manifolds due to H20 leaks }\end{array}$ & Improve reliability & Complete \\
\hline
\end{tabular}




\begin{tabular}{|c|c|c|}
\hline Machine/Activity & Goal & Status \\
\hline \multicolumn{3}{|l|}{ Main Injector } \\
\hline $\begin{array}{l}\text { Install MI-30 collimation system add } 2 \\
\text { collimation masks }\end{array}$ & $\begin{array}{l}\text { Increased intensity to NuMI: } \\
\text { control of losses to allow } \\
\text { implementation of multi-batch slip } \\
\text { stacking. A Proton Plan activity. }\end{array}$ & Complete \\
\hline \multicolumn{3}{|l|}{ Recycler } \\
\hline Pelletron maintenance & Improved reliability & Complete \\
\hline \multicolumn{3}{|l|}{ Tevatron } \\
\hline Unroll Tevatron dipoles & Global coupling reduction & $\begin{array}{l}\text { Magnet alignment re-checked and aligned } \\
60 \text { magnets. }\end{array}$ \\
\hline Repair vacuum & Improved reliability & $\begin{array}{l}\text { Nine (of 24) houses were warmed up. } \\
\text { Multiple leaks were identified and repaired. } \\
\text { Complete }\end{array}$ \\
\hline Check alignment of low-beta magnets & Improve operability & Alignment OK; didn't need to be moved. \\
\hline \multicolumn{3}{|l|}{ NuMI } \\
\hline Repair Horn 2 water leak & Operability & Complete \\
\hline $\begin{array}{l}\text { Replace water connections on } 6 \text { injection } \\
\text { magnets }\end{array}$ & Improved reliability & Complete \\
\hline Target replacement & Increase neutrino production rate & Complete \\
\hline Replace hadron detector in MINOS & Improve diagnostics & Complete \\
\hline \multicolumn{3}{|l|}{ NOvA } \\
\hline $\begin{array}{l}\text { Civil construction at MI-39 and MI-14 for } \\
\text { installation of kicker cable conduits }\end{array}$ & $\begin{array}{l}\text { Laboratory upgrade for NOvA } \\
\text { project }\end{array}$ & Complete \\
\hline Install 7 kickers & $\begin{array}{l}\text { Laboratory upgrade for } \mathrm{NO} v \mathrm{~A} \\
\text { project }\end{array}$ & Complete \\
\hline \multicolumn{3}{|l|}{ Infrastructure Maintenance } \\
\hline Maintenance of the feeder system & Reliability risk reduction & Complete \\
\hline Maintenance at both Fermilab substations & Reliability risk reduction & Complete \\
\hline
\end{tabular}

\section{$\underline{\text { E-830 / Collider Detector at Fermilab (CDF) }}$ (M. Casarsa, P. Schlabach)}

During FY 2009 a total integrated luminosity of $1.9 \mathrm{fb}^{-1}$ was delivered to the CDF detector, of which $1.6 \mathrm{fb}^{-1}$ was recorded to tape. At the end of FY 2009, a total of $6.9 \mathrm{fb}^{-1}$ had been delivered to the experiment in Run II (Figure 4). Overall, the CDF detector has been operating with good live-time and no significant issues, even at the highest instantaneous luminosities. The total data-collection efficiency was $85 \%$, including dead time associated with trigger acceptance, operational inefficiencies (e.g. starting and stopping runs), and downtime from detector problems. 


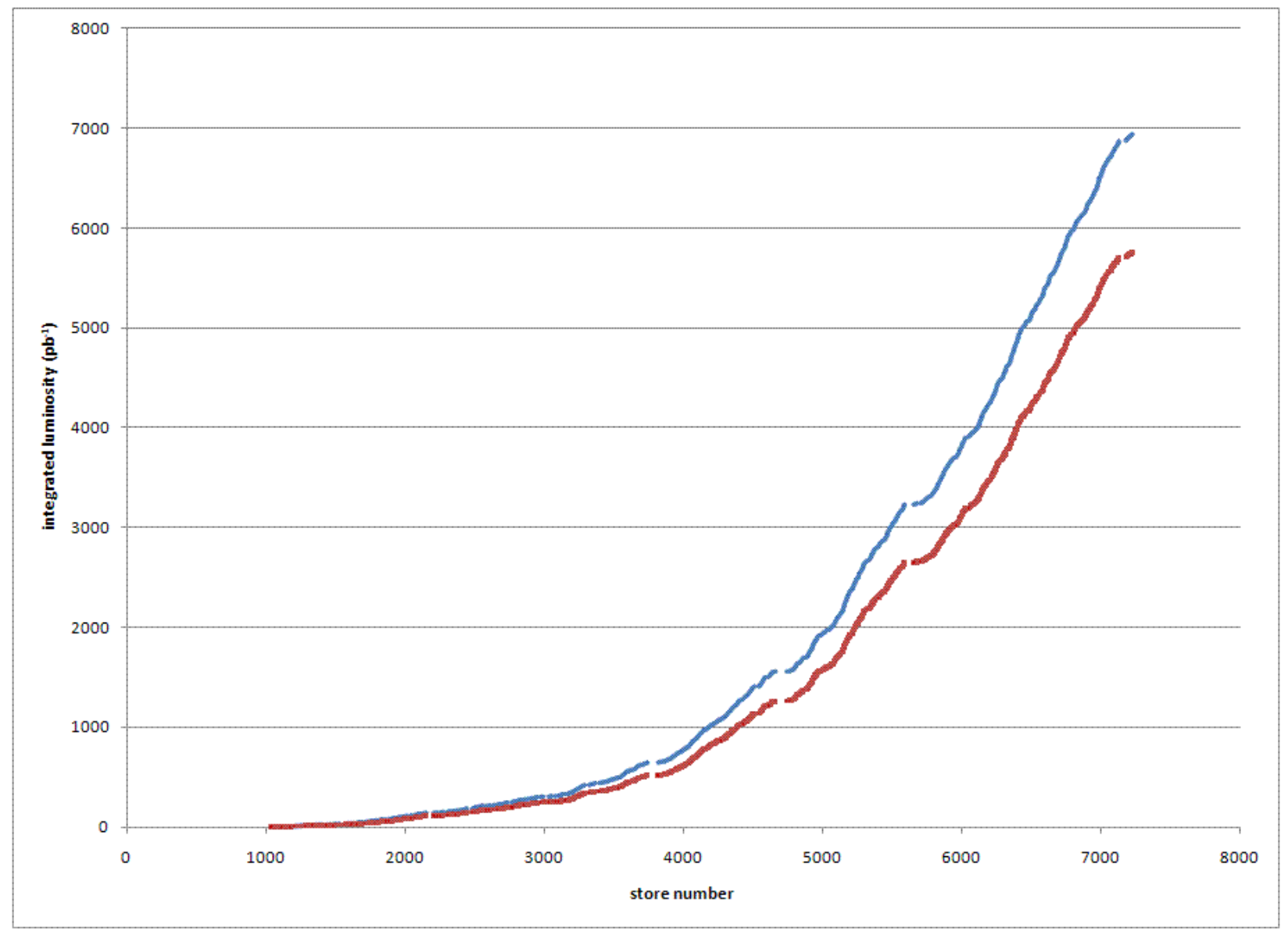

Figure 4. Delivered and recorded luminosity at CDF in Run II.

The Tevatron initial instantaneous luminosities were steadily around $3 \times 10^{32} \mathrm{~cm}^{-2} \mathrm{sec}^{-1}$. The median, average and maximum were 3.0, 2.8 and $3.5 \times 10^{32} \mathrm{~cm}^{-2} \mathrm{sec}^{-1}$. The CDF trigger is coping well with the higher instantaneous luminosities due to the upgrades of the past several years (L2 stereo tracking, L2 calorimeter, L1 missing energy, L3 muon code). Towards the end of the year, CDF began parasitic testing of a proposed upgrade to the online displaced vertex trigger. This consolidates track fitting from 16 boards to 1 board on which more modern processors fit tracks faster. The upgrade is intended to increase efficiency and improve purity while potentially allowing lower-impact parameter and transverse momentum cuts and increased acceptance for forward tracks.

There were two planned accelerator shutdown periods during the fiscal year: one-week at the beginning and 11 weeks at the end. As can be seen by the data-taking efficiency shown in Figure 5, the short shutdown had minimal effect while at the end of the fiscal year CDF was returning to the efficient running enjoyed before the shutdown. 


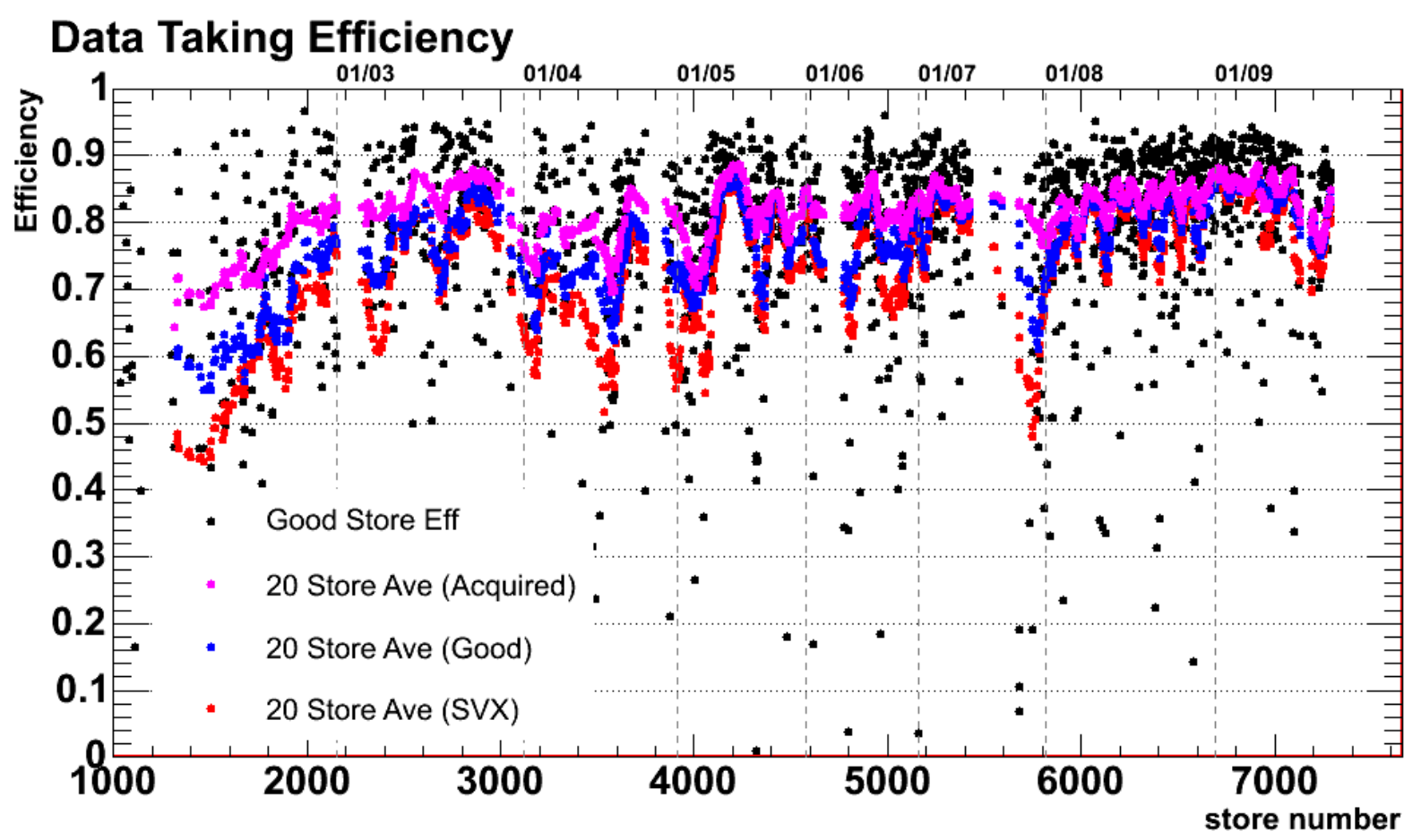

Figure 5. Data-taking efficiency for CDF in fiscal years 2001-2009.

During the one-week shutdown CDF opened both end plugs and performed preventive maintenance aimed at mitigation of potential HV problems in the COT. No major HV problems occurred during the rest of the year.

During the long shutdown a number of major jobs were accomplished. CDF opened the end plugs and did an extensive investigation of the ISL cooling on the east side. The cooling had deteriorated somewhat since it was fixed in late FY 2007. While a number of leaks were found and sealed, and flow improved in several locations, the system ended up where it started as far as overall leak rate goes. The leak or leaks that dominate were not fixed. There was also extensive work on refurbishing silicon power supplies. Between the improved flow and supply fixes, five additional ladders of the system were recovered.

During the extended period with the plugs open, CDF was able to access the plug source drives for maintenance. Three sources were removed and sent to Purdue for repairs, and many adjustments to the drive system were made. This allowed the sources to reach many areas of the detector which had been unreachable for years. A full source-testing of the detector was performed, and the high voltage was adjusted to compensate for the aging of the detector.

Other work performed included preventive maintenance and upgrades to the electrical and mechanical infrastructure. Additional work on the COT HV during the bore access recovered more wires. CDF patched and upgraded software on the online machines, and replaced 64 nodes of the L3 farm with new ones. CDF also tied in a new emergency generator. Finally, over 200 people had a chance to visit the hall and admire the detector. 
There were no major changes in shift operations. The number of offshore institutions participating in remote data-quality monitoring is now four since Padova (Italy) came online, joining Pisa, Tsukuba (Japan), and KISTI (Korea). CDF continued to move responsibilities around as institutions and people gradually leave. The process is gradual; CDF moved a few systems per year for the past couple of years, and these adjustments were accomplished without issue.

$\underline{\text { E-823 / DZero (DZero) }}$ (D. Denisov, G. Ginther, S. Gruenendahl, W. M. Lee, S. SoldnerRembold)

The accelerator complex delivered an integrated luminosity of $1.91 \mathrm{fb}^{-1}$ to DZero during FY 2009. DZero recorded $1.75 \mathrm{fb}^{-1}$, corresponding to an operating efficiency of $91.6 \%$ during that time period. Figure 6 illustrates the detector efficiency as a function of time during Run II. The FY 2009 performance represents the best yearly operating efficiency achieved by DZero to date. These achievements were made possible by increases in instantaneous and integrated luminosities delivered by the accelerator complex and operational improvements in the detector.

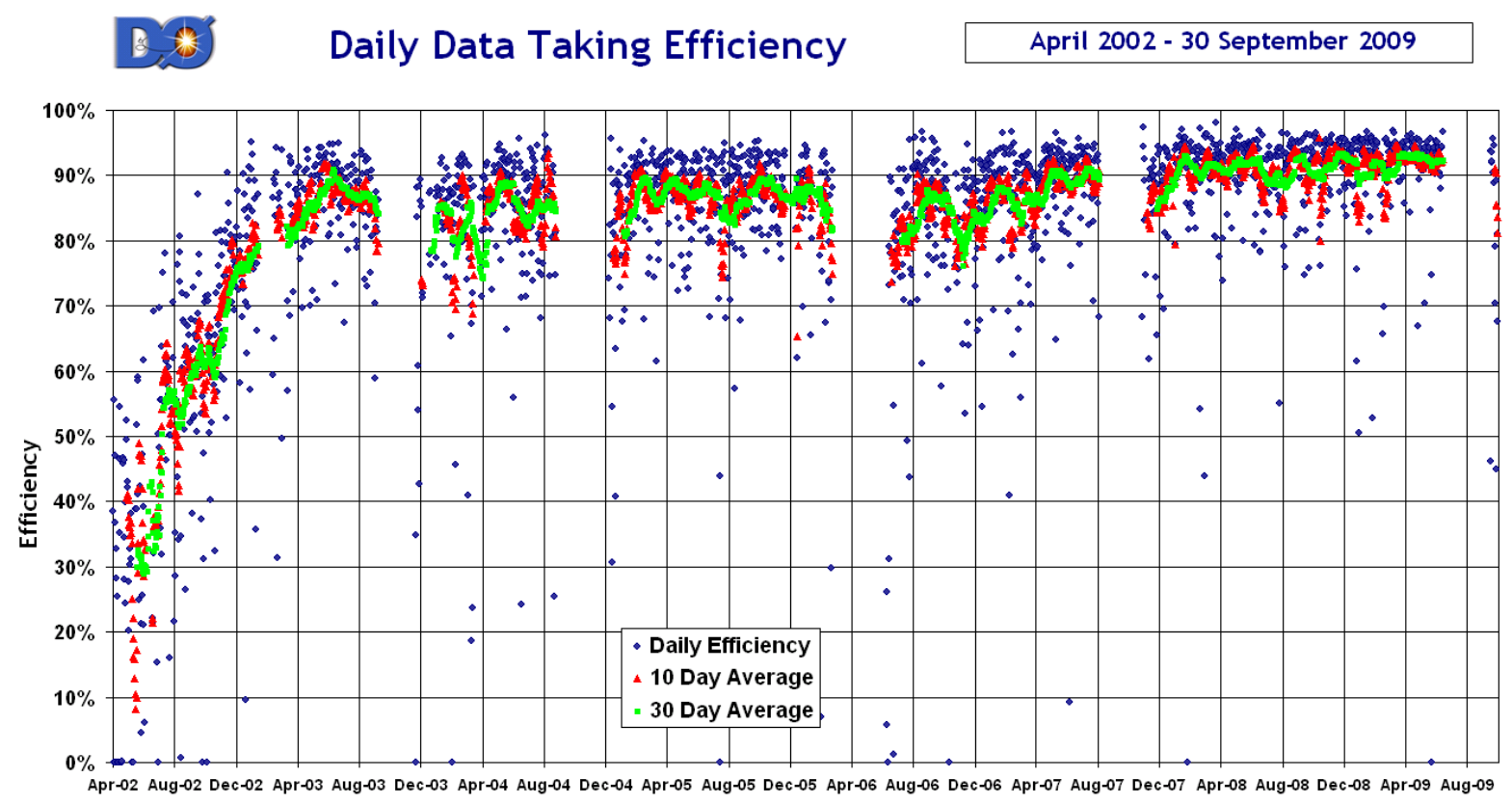

Figure 6. DZero data-taking efficiency as a function of time during Run II. The blue dots represent a daily average, the red triangles represent a 10-day average, and the green squares represent a 30-day average.

As a result of an intensive effort, DZero revived more than 5\% of the Silicon Microstrip Tracker barrel readout channels during the one-week 2008 shutdown, and then rapidly resumed smooth operations upon completion of that shutdown. DZero used the 11-week-long 2009 shutdown access time to perform routine maintenance and introduce additional improvements and refinements. The luminosity monitors were replaced due to radiation damage to the scintillators. A substantial effort was undertaken to study, and where possible, recover the remaining non-functional Silicon Microstrip Tracker readout channels. More than 5\% of the 
barrel channels were returned to service during this process, and there were corresponding gains in the operational disk channels. Liquid argon levels in the calorimeters can slowly decrease due to pressure differences between the cryostats and the storage dewar. Consequently, the liquid argon levels were topped off in the south End Cap Calorimeter and the Central Calorimeter. Significant effort was invested in further enhancing the performance of the Inter Cryostat Detector by replacing 30 photomultiplier tubes and 25 high-voltage pods. The muon systems were calibrated and the firmware was refreshed. The Central Track Trigger equations were reevaluated to take into account updated alignment input, and studies aimed at further enhancements were initiated. The operating systems for the online cluster were upgraded, as were the fileservers. The disk devoted to short-term storage of the data was also upgraded. Safety system tests, single channel repairs, and routine and preventive maintenance were completed to prepare the detector for the long operating period from 2009 through 2011.

As illustrated in Figure 6, the start-up of the DZero detector after the 2009 summer shutdown went well. The detector is performing well. DZero was recording physics-quality data within two hours of the start of the first post-shutdown store. Special triggers to gather calorimeter calibration data were collected in parallel with the standard physics suite to verify the calorimeter calibration.

DZero continued efforts to streamline operations in the face of increasing instantaneous luminosity, and continued to refine its operations in an effort to improve efficiency. During the past year, streamlining of the begin-store and end-store activities resulted in a reduction in these transition times by about three minutes each, contributing to improved efficiency (from the begin-store activities) and increased opportunity to record data (from the end-store related activities). The number of run transitions during stores was reduced, and the downtime required to execute a run transition was reduced also. The detector continued to operate reliably, and recorded data at record rates (due to the excellent performance of the Tevatron Collider). The operating voltages of the Silicon Microstrip Tracker were adjusted to maintain optimal performance, and monitoring of the impact of radiation damage continued. The results of this monitoring indicate that by the end of Run II, as anticipated, it will no longer be possible to fully deplete all of the Layer 1 silicon. Layer 0 was installed during the 2006 shutdown, in part to compensate for this anticipated degradation in performance. In addition to ongoing efforts to address remaining sources of dataflow interruptions, there were continuing efforts to improve monitoring tools, and the guidance, training and documentation provided for the shift crews that operate the detector around the clock.

DZero continued to optimize its trigger suite to facilitate routine and efficient operation with initial luminosities above $3 \times 10^{32} \mathrm{~cm}^{-2} \mathrm{sec}^{-1}$. The trigger list is designed to efficiently handle initial luminosities up to $3.5 \times 10^{32} \mathrm{~cm}^{-2} \mathrm{sec}^{-1}$ without prescaling high-priority high- $\mathrm{p}_{\mathrm{t}}$ physics triggers. As of the end of FY 2009, the record initial luminosity at DZero was $3.5 \times 10^{32} \mathrm{~cm}^{-2} \mathrm{sec}^{-1}$, and the trigger and detector performed well under these conditions.

The average operating efficiency of the detector continued its gentle rise, and is at $88.4 \%$ for the entire Run II data sample. The integrated luminosity recorded during FY 2009 was $9 \%$ larger than during FY 2008, which was the previous best performance. This record performance was realized in spite of the fact that there were ten more weeks of Collider shutdown during FY 2009 than in FY 2008. As illustrated in Figure 7, the total recorded integrated luminosity increased by $40 \%$ to $6.16 \mathrm{fb}^{-1}$. 


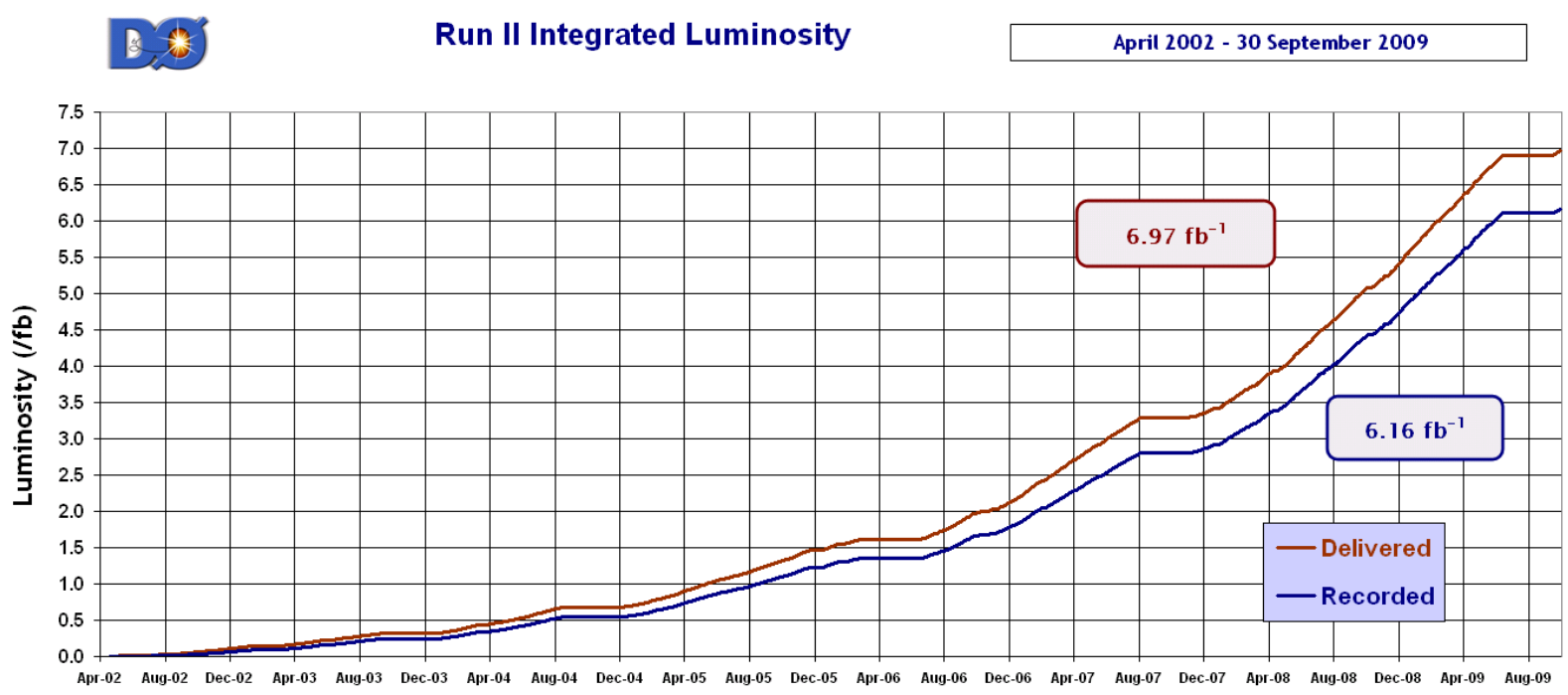

Figure 7. Delivered (red line) and recorded (blue line) luminosity at DZero as a function of time during Run II.

With the significant improvements in the antiproton stacking rate achieved by the Fermilab Accelerator Division and the ongoing efforts to maintain performance and reduce shot setup time, the delivery of more than $12 \mathrm{fb}^{-1}$ to each of the collider detectors before the end of FY 2011 looks achievable. In addition, DZero continues to publish papers at an average rate that exceeds three papers a month. Given the smooth and efficient operation of the DZero detector, the collaboration is well positioned to take full advantage of increasing luminosity and to continue its multifaceted exploration of the high-energy physics frontier.

NuMI / E-875 Main Injector Neutrino Oscillation Search (MINOS) (C. White, R. Plunkett)

$\underline{N u M I}$

NuMI began FY 2009 with steady and excellent beam running. However, on November 17, 2008, after more than 25 million beam pulses, the strip-line on Horn 2 developed a ground fault. Two washers holding ceramic spacers between strip-line sections apparently became brittle and failed. When these washers failed the bolts were able to move, up to about 0.25 inches. Continued movement from repeated pulsing caused the nuts to eventually walk-off. This also allowed the strip-line segments to repetitively flex from horn pulsing, which produced a fracture at the junction box just below the remote clamp. The Horn 2 replacement was completed on December 12. Additional tritium mitigation dehumidification was added in the absorber area in parallel with the horn replacement. Maintenance and repairs were also conducted on target pile dehumidification equipment. High-intensity NuMI operations were quickly restored and steady running continued through June 13, 2009 (the beginning of the extended accelerator complex shutdown), as shown in Figure 8. Several records were achieved during this period: $76.2 \times 10^{17}$ protons on target for the week beginning March 30 , and $12.2 \times 10^{17}$ protons on target delivered on April 14, part of the record $78.7 \times 10^{17}$ protons on target for the week beginning April 13 . 


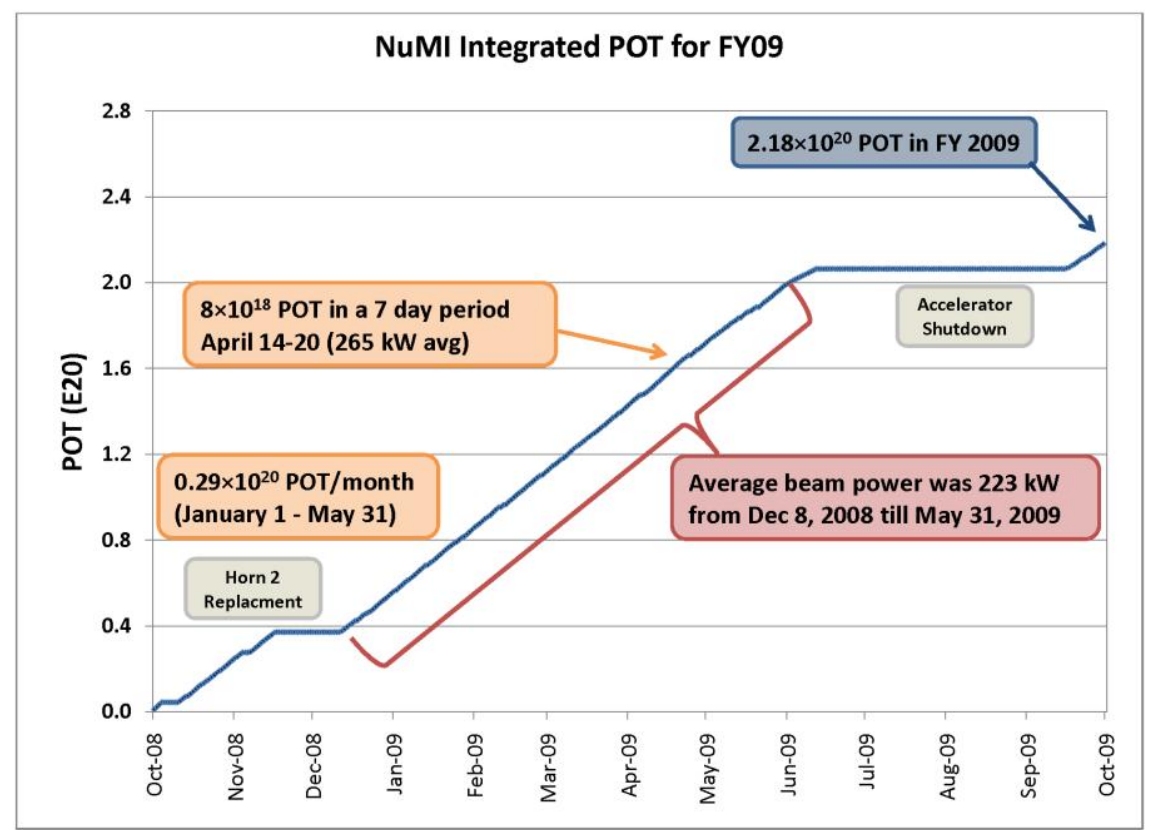

Figure 8. NuMI's integrated protons-on-target for FY 2009. A total of $2.18 \times 10^{20}$ protons were delivered during the fiscal year. Except for a brief downtime to replace Horn 2, the year was notable for uniform and consistent beam.

A number of significant improvements were made to the NuMI beamline during the extended shutdown:

1. Since 2005 , both horns have been replaced. It is anticipated that the horns will need to be serviced and/or replaced in the future. To facilitate this work, a rail system was designed and installed, complete with carts and coffins to move broken horns and strip-line blocks out of the target hall.

2. The $\mathrm{C} 0$ building has been designated as a storage and repair facility for NuMI horns, and is undergoing conversion.

3. MINOS data collected during the past year indicates that the neutrino yield per protonon-target had been declining. The reason for the decline was attributed to degradation of the target. A new target was constructed, and was installed during the shutdown.

4. In anticipation of switching to antineutrino running, the horn power supplies were upgraded to provide reverse polarity running.

5. The hadron monitor was replaced due to radiation damage.

6. Movable aluminum absorber blocks were installed in front of each muon monitor to study delta-ray production and to better understand and predict delta-ray backgrounds during normal operations.

Beam returned to NuMI on September 11, 2009, but was restricted to low intensity for several days for a series of target scans (to verify the installation and alignment of the new 
target). Normal operations resumed on September 15 in neutrino mode. NuMI collected $1 \times 10^{19}$ protons on target to validate the new target before switching to antineutrino mode on September 29, 2009. A total of $2.18 \times 10^{20}$ protons-on-target was delivered for NuMI in FY 2009.

\section{MINOS}

The MINOS experiment continued efficient operation in FY 2009. Both Near and Far Detectors operated at extremely high efficiency, 99\% live-time with beam for the statisticslimited Far Detector, and 98\% overall (including scheduled shutdown maintenance). The Near and Far Detectors are robust, requiring only modest ongoing maintenance and upgrades (e.g. replacement of UPS batteries, network upgrades, etc). Typically, the Far Detector has no dead channels, with minor problems repaired rapidly as they appear. The Far Detector required only minimal preventive maintenance during the extended accelerator shutdown, and remained live throughout the summer collecting cosmic-ray data. The Near Detector electronics also continued to perform in a steady and consistent fashion resulting in a similar problem-free readout. The Near Detector's downtime was dominated by infrastructure work related to new experiments (MINER $v$ A and ArgoNeuT) moving into the Near Detector Hall. To provide systematic controls on the magnetic-field strength, regular field reversals became a feature of Near Detector operations in FY 2009, with the field changing sign every two weeks. This mode of operation may continue in FY 2010. As with the Far Detector, only preventive maintenance was performed on the Near Detector during the extended shutdown, although the Near Detector was turned off for five weeks spanning a series of scheduled power outages at Fermilab. The end of the summer shutdown also marked a new beginning for MINOS. Following the validation run for the new NuMI target, the MINOS detectors were configured for antineutrino running by reversing the magnetic field at the Far Detector. MINOS anticipates operating in this mode until March 2010.

The Near Detector Hall is now shared with several experiments with a subsequent impact on MINOS Near Detector operations. Ongoing construction activities occasionally create undesirable conditions, such as increased electronic noise in the Near Detector electronics, necessitating turning off the Near Detector at times. Another ongoing issue is heat load. Ground water seeping into the Near Detector Hall is pumped through heat exchangers before being pumped to the surface. As the Hall matures, the rate of seepage has decreased, reducing the capacity of the Near Detector Hall cooling system. Heat load is increasing as cooling capacity decreases. Long-term infrastructure improvements may be required, and discussions of technical solutions are ongoing.

The most recent MINOS update on the $v_{2} \leftrightarrow v_{3}\left|\Delta \mathrm{m}^{2}\right|$ oscillation parameter appeared in publication in September 2008. A summary is included here for completeness. It was based on an exposure of $3.36 \times 10^{20}$ protons on target, as shown in Figure 9 and published in Phys. Rev. Lett. 101:131802 (2008). It is based on less than half of the current data set. Ongoing analysis of the full data set will continue to improve the knowledge of the $v_{2} \leftrightarrow v_{3}\left|\Delta \mathrm{m}^{2}\right|$ oscillation parameter. The oscillation parameters are now measured to be $\Delta \mathrm{m}^{2}=2.43 \pm 0.13 \times 10^{-3} \mathrm{eV}^{2}$ and $\sin ^{2} 2 \theta=1.00_{-0.05}^{+0.00}$ (one sigma errors). MINOS data disfavors non-standard $v_{\mu}$ disappearance models such as neutrino decay (by 3.7 $\sigma$ ) and decoherence (by 5.7 $\sigma$ ). 


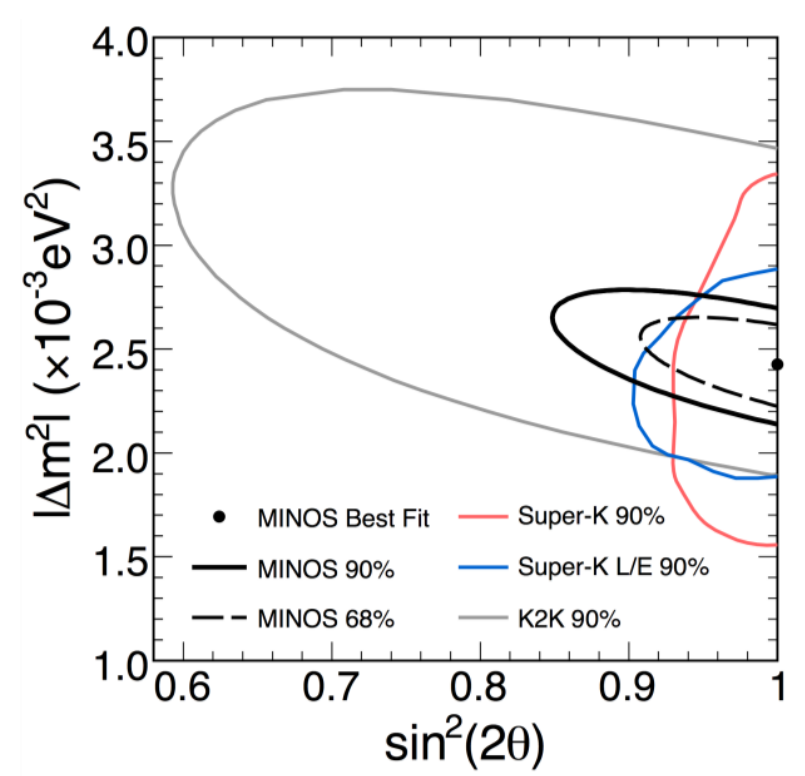

Figure 9. The allowed region in parameter space from the analysis of charged-current $v_{\mu}$ interactions in MINOS resulting from $3.36 \times 10^{20} \mathrm{NuMI}$ protons-on-target, compared to previous experiments.

To check if the observed $v_{\mu}$ disappearance might involve a fourth, sterile neutrino flavor, the rate of Neutral Current (NC) interactions can be compared between the Near and Far Detectors. Such a sterile neutrino would undergo no NC interactions while any of the three known flavors would. An exposure of $2.46 \times 10^{20}$ protons-on-target has been used to make a comparison of the Far Detector spectrum with expectations derived from the observed highstatistics Near Detector data. The data are consistent with no sterile neutrino participation, with a measurement of the fraction of sterile participation $\mathrm{f}_{\mathrm{s}}<0.68$ at $90 \%$ confidence level. The result was published in FY 2009, Phys. Rev. Lett. 101:221804 (2008). Analysis of a larger data set $\left(3.18 \times 10^{20}\right.$ protons-on-target) was completed in FY 2009 and a publication to be submitted to Physical Review D was circulated for final collaboration approval, with submission expected in early FY 2010.

The MINOS detector was optimized for charged-current neutrino interactions that produce muons. While it is more difficult to observe electrons, it is possible. This past year the Collaboration completed the first analysis of electron appearance in the MINOS Far Detector, culminating in a plethora of conference and seminar presentations. Based on an exposure of $3.2 \times 10^{20}$ protons-on-target, MINOS observes $35 v_{\mathrm{e}}$ charged-current events with an expected background of $27 \pm 5$ (stat) \pm 2 (syst), as shown in Figure 10. A paper has been submitted to PRL. An updated analysis using approximately $7 \times 10^{20}$ protons-on-target will be released in 2010 . 


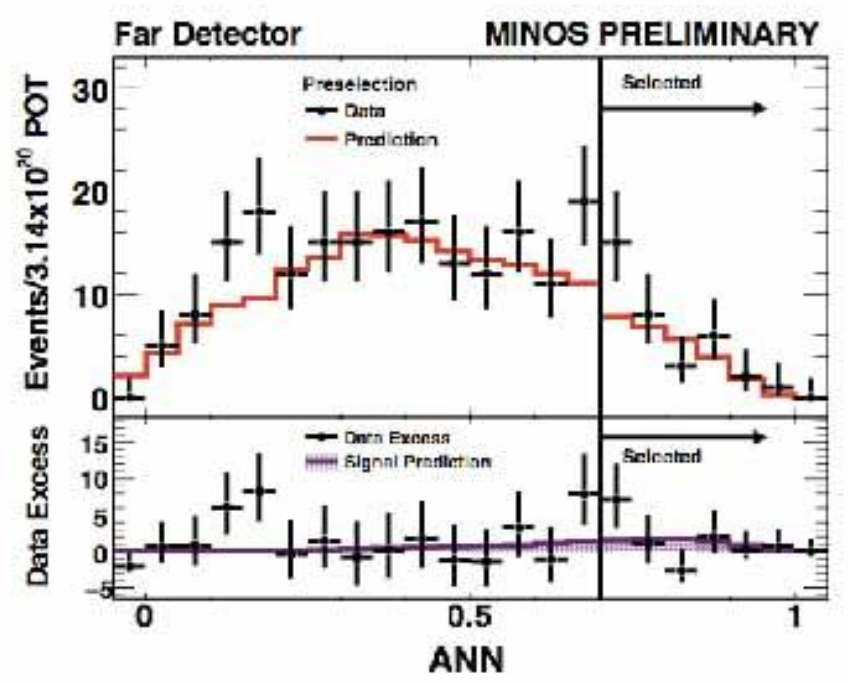

Figure 10. Distribution of the Artificial Neural Network (ANN) selection variable for data and MC events in the Far Detector. Black points show data with statistical error bars. The red histogram shows the background expectation. Below, the purple shaded histogram shows the $v_{\mathrm{e}} \mathrm{CC}$ prediction by the oscillation hypothesis compared to the data excess (black points).

An analysis of the rate in the Far Detector of antineutrino events in the neutrino beam, using approximately half of the data set accumulated through FY 2009, was completed during this period. Antineutrinos are a background which appears in the NuMI neutrino beam at approximately the $6 \%$ level. Muons from the charged-current interactions of antineutrinos are selected using the magnetic field of the Far Detector. MINOS presented preliminary results from this analysis, in which 42 events are observed, $1.9 \sigma$ fewer than the expectation of 58. As with the other analyses, the antineutrino sample has approximately doubled since this work. An analysis of the full sample will reduce the statistical uncertainties which dominate.

In addition to the oscillation-related analyses, MINOS published in FY 2009 a paper on "Sudden stratospheric warmings seen in MINOS deep underground muon data", which appeared in Geophys. Res. Lett. 36:L05809 (2009). Also, "Observation of muon intensity variations by season with the MINOS far detector" was submitted for publication in Phys. Rev. D. Collaboration members also published two technical papers.

E-944 / MiniBooNE (S. Brice, R. Van de Water)

FY 2009 Booster Neutrino Beam running for MiniBooNE was entirely in antineutrino mode. During this period, MiniBooNE collected data from $1.2 \times 10^{20}$ protons on target. Added to previous antineutrino running, this allowed MiniBooNE to reach its goal of a total of $5 \times 10^{20}$ protons on target in antineutrino mode by the 2009 shutdown. First antineutrino oscillation results have been published (arXiv:0904.1958 [hep-ex], Phys. Rev. Lett. 103:111801 [2009]), and new updated results with the recently collected data were presented publicly at the fall 2009 PAC meeting. MiniBooNE will run for another $2 \frac{1 / 2}{2}$ years to double antineutrino statistics for a 
total of $10 \times 10^{20}$ protons on target. This will help to improve the statistics-limited antineutrino oscillation measurement.

Figure 11 shows the weekly performance of the Booster Neutrino Beam since the start of running in 2002. The FY 2009 antineutrino running is shown in the shaded regions. The performance of the Booster during FY 2009 was steady, with beam uptime over 90\% and average weekly delivered protons of $0.35 \times 10^{19}$ protons on target/week. The weekly protons-ontarget spike in October 2008 was when NuMI was down for three weeks to repair the horn. MiniBooNE can take advantage of such unscheduled peak protons-on-target periods due to its high reliability and uptime.

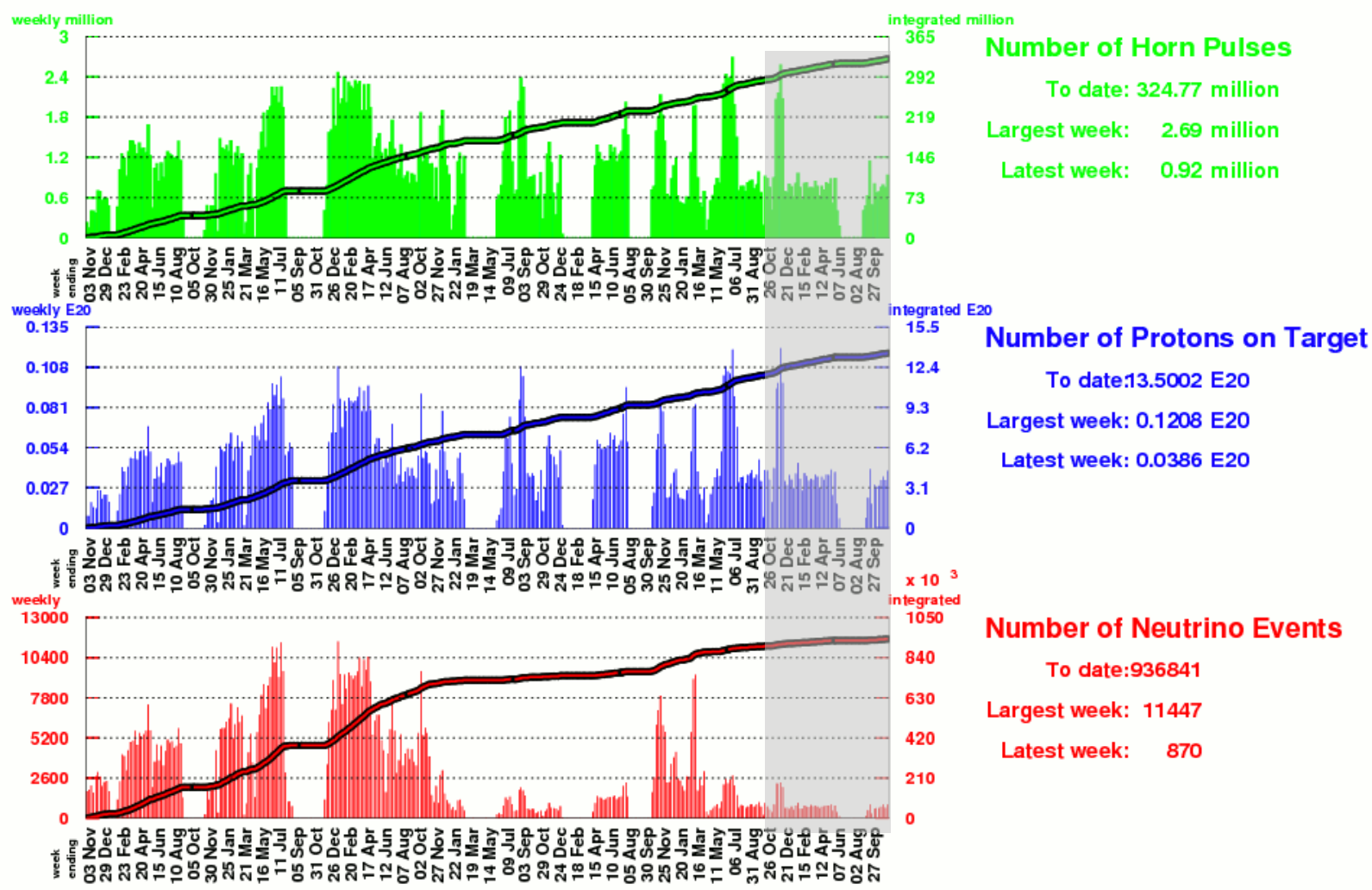

Figure 11. MiniBooNE weekly beam summary since the start of operations in 2002. The FY 2009 antineutrino running is shown in the grey-shaded regions. Antineutrino event rates are down by a factor four relative to neutrino running due to decreases in flux and cross sections.

Figure 12 shows the detector energy scale stability as measured by Michel electrons over the entire run from 2002 to present. The fractional deviation (bottom plot) is within 1\% during this time, indicating the high level of stability of the detector oil, photomultiplier tubes and electronic digitization response. Due to this stability, MiniBooNE is confident, despite the age of the detector, that high quality antineutrino data can be collected to reach the goal of $10 \times 10^{20}$ protons on target in antineutrino mode. 

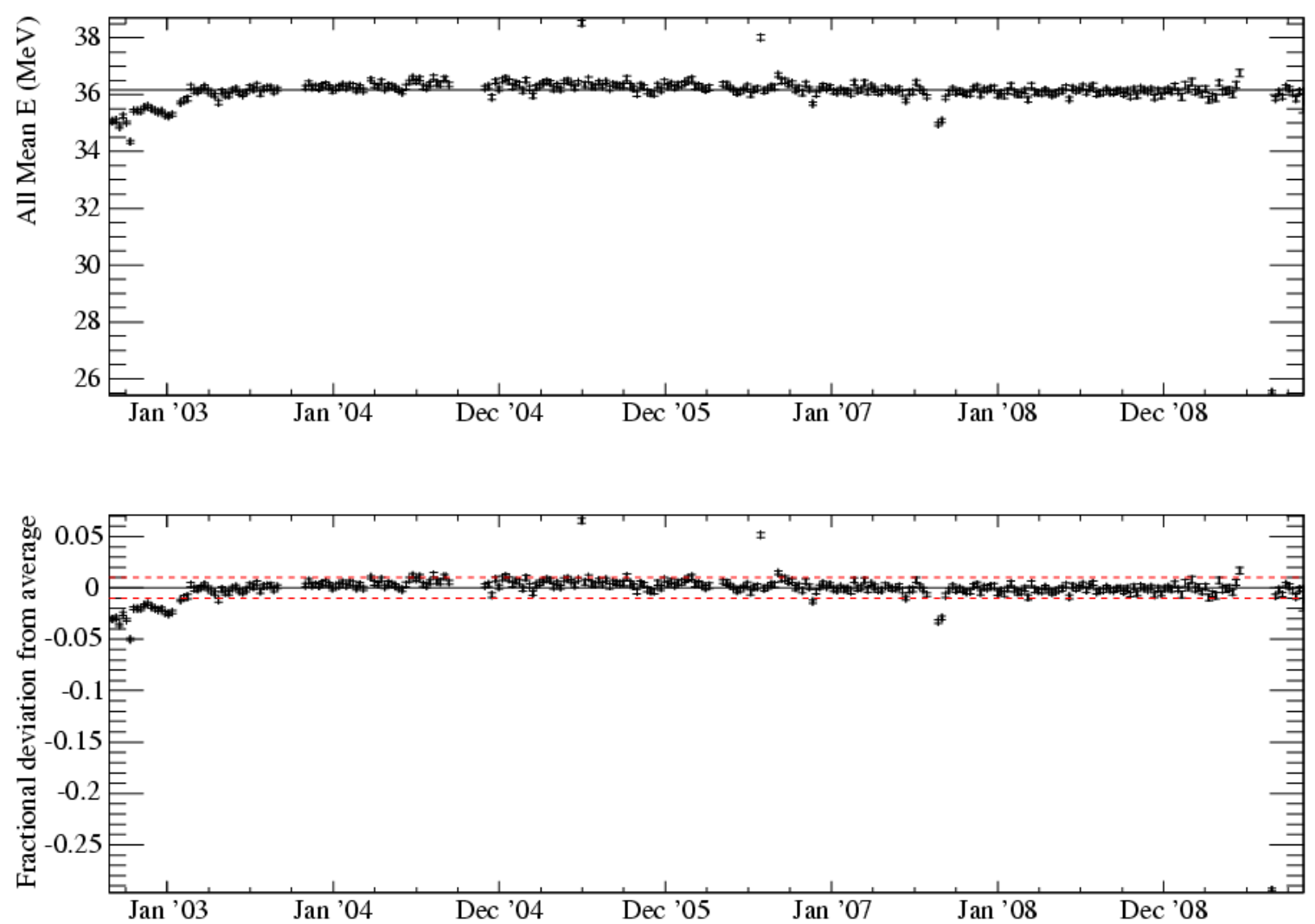

Figure 12. MiniBooNE Michel electron energy scale (top) since the start of running in 2002. The bottom plot shows the fractional deviation of the energy scale from average. The deviation is within $1 \%$ for the duration of the run, demonstrating a high level of detector response stability. The larger energy response difference around January 2003 is during detector commissioning. This data is not included in any physics analysis.

Another important aspect of continued running of MiniBooNE is the reliability of the beamline and horn. For the FY 2009 antineutrino run period, the combined beamline and detector uptime was over 95\%. The most crucial element, the horn, has collected over 230 million pulses, surpassing the first horn, which failed four years ago with 94 million pulses (both these numbers are world records). This second horn continues to run flawlessly, with no sign of problems. In the unfortunate event of failure, a spare horn and target are available. It would require three weeks to install the replacement system.

Fixed-Target Switchyard $120 \mathrm{GeV}$ (SY120) and MTest (R. Coleman, W. Kissel, C. Moore, E. Ramberg)

\section{Switchyard $120 \mathrm{GeV}$ Extraction from Main Injector in FY 2009}

The Fermilab test beam originates from the resonant extraction of one Booster batch inside the Main Injector (MI). This batch usually consists of 20-60 RF 'buckets', with buckets 
separated by $19 \mathrm{nsec}$. Thus the batch is about 1 microsecond long. The time required for a particle to transit the full circumference of the MI is about 11 microseconds. The batch is accelerated to $120 \mathrm{GeV}$, circulated around the MI, and is slowly extracted over a time period of a few seconds as a quadrupole gradually increases in field strength.

The length and duty cycle of the spill is determined by the Accelerator Division (AD), with guidance from the Program Planning Office. For FY 2009 there was a single 4-second-long spill per minute, for a maximum of up to 14 hours per day. The AD has set up a procedure for changing from this four-second spill to a one-second spill. This shorter spill can then be delivered more frequently for commissioning purposes, and for those groups which are limited by data-acquisition buffers. The number of SY120 pulses with beam in FY 2009 was 34,794.

In FY 2009 the AD completed commissioning a "ping" beam extraction from the Main Injector, where the extraction quadrupole is brought close to its nominal strength, and then pulsed with current over a very short $(1-5 \mathrm{msec})$ period. A large fraction of the beam then spills out in that short period. This can then be repeated at periodic intervals as the Main Injector holds its flattop. If the ping is $1 \mathrm{msec}$ long and the interval between pings is $200 \mathrm{msec}$, this then replicates the macroscopic duty cycle of the proposed International Linear Collider (ILC). This makes Fermilab's MTest the only facility able to deliver high-energy particles with this structure. Figure 13 shows oscilloscope evidence for this pinged extraction.

\section{First Pings to MTest}

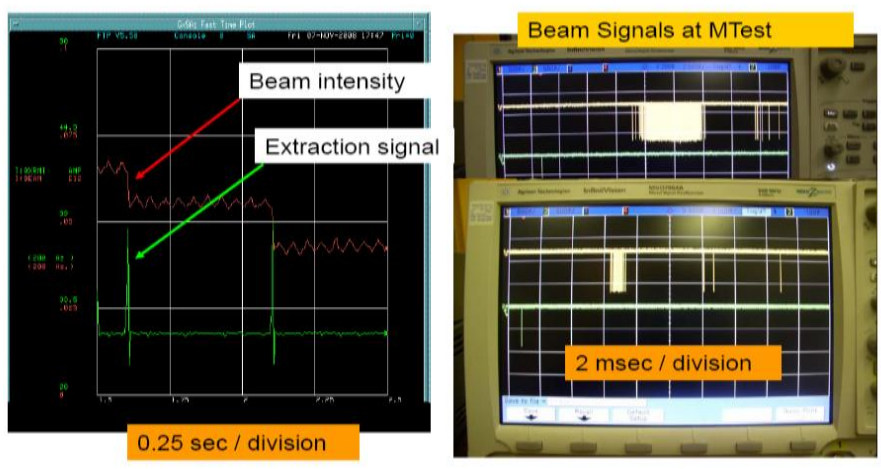

Figure 13. Two scope traces of scintillator hits during pinged MI extraction.

The extracted $120 \mathrm{GeV}$ primary proton beam has an approximate $0.3 \%$ momentum spread and can be focused to a $7 \mathrm{~mm}$ rms spot size at the user facility (described below). In addition to delivering primary beam to users, the facility can target this beam on a secondary target and deliver lower-energy beams in the energy range of 1-66 GeV. There is a 1-10\% momentum spread with approximately $2-5 \mathrm{~cm}$ rms spot size for secondary beams of lower momenta.

During 2007, the MTest secondary beamline was optimized for low-energy particle production. In addition to the ability to run at much lower energy, the new beamline had several other improvements in instrumentation and tuning facilities which improved general operations. 
The ILC CALICE calorimeter group (T-978) was particularly interested in this low-energy pion capability. In FY2009, CALICE utilized the secondary beam for four weeks of tests. Tunes for the beamline were optimized for the following momenta for negatively-charged beams: 1, 2, 3, $4,6,8,10,12,15,20$, and $30 \mathrm{GeV} / \mathrm{c}$. In addition, it was observed that inserting a movable absorber located in the user area and tuning for positive $32 \mathrm{GeV} / \mathrm{c}$ secondary beam maximizes the rate $(\mathrm{kHz}$ level) of a wide-band muon beam for users.

\section{The Test Beam User Facility}

The test beam user facility gives users from around the world an opportunity to set up their particle detectors in a variety of particle-beam types. A plan view of the facility is shown in Figure 14. The web site for the MTest facility can be found at www-ppd.fnal.gov/MTBF-w.

\section{MT6 Test Beam User Areas}

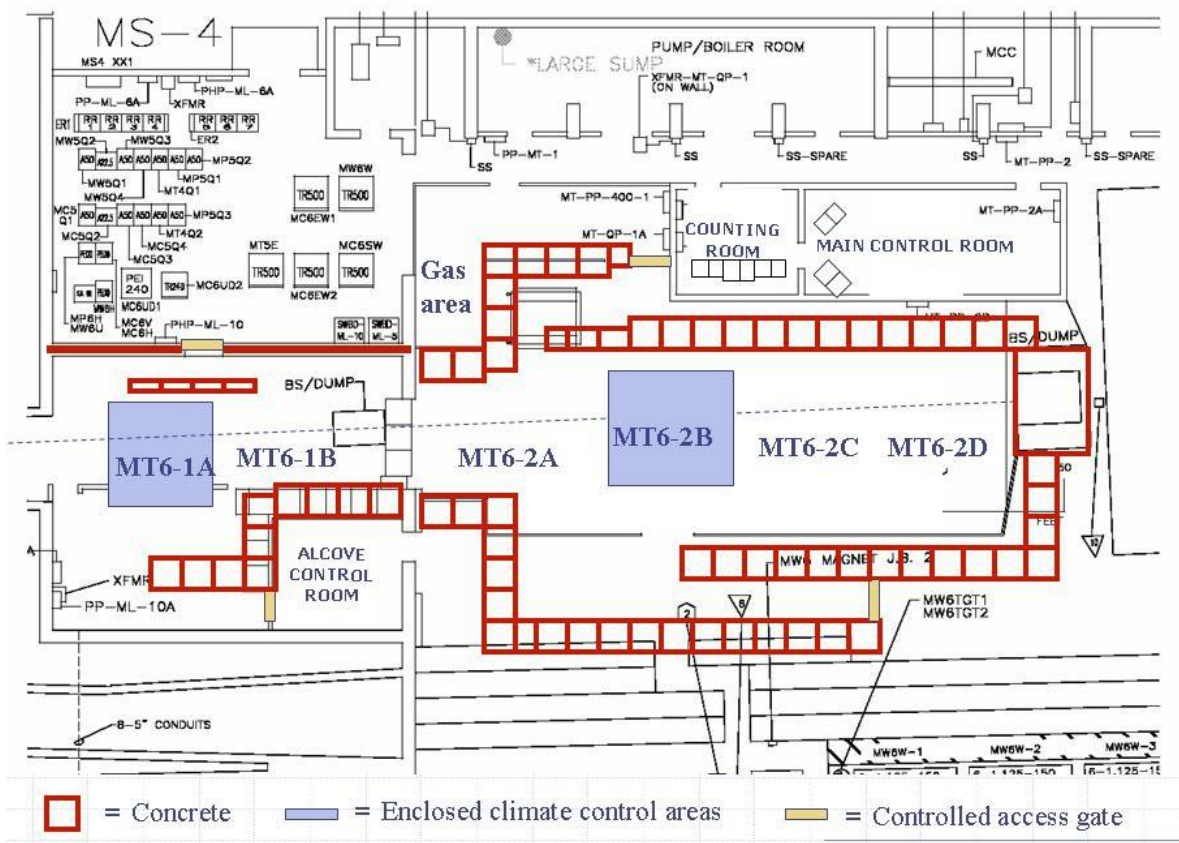

Figure 14. Plan view of the Meson Test Beam Facility.

\section{Research performed at the MTest Facility in FY 2009}

Each test-beam experiment is required to prepare a Memorandum of Understanding with the Laboratory, in which the beam, infrastructure, and safety requirements are spelled out in detail. Two new experiments were approved and took data during FY 2009. An additional five experiments that took data in FY 2008 returned to take data in FY 2009. These seven experiments are listed in Table 3. These experiments installed equipment or took data for a total of 14 weeks, out of the 39 available weeks during the year. In addition, there were several weeks devoted to major installations of new equipment and several weeks devoted to beam studies for the facility. 
Table 3. MTest experiments performed in FY 2009.

\begin{tabular}{|l|l|}
\hline Test & Description \\
\hline & \\
\hline T-992 & Rad hard sensors for SLHC (New) \\
\hline T-988 & AIRFLY - N2 fluorescence (New) \\
\hline T-979 & Ultra Fast Timing \\
\hline T-978 & CALICE - ILC Calorimetry \\
\hline T-977 & MINERvA Experiment \\
\hline T-971 & LHCb Silicon Detector Upgrade \\
\hline T-953 & U. Iowa Cerenkov Light Tests \\
\hline
\end{tabular}

The CALICE experiment (T-978) is a collaboration among a large number of mostly European groups, headed in large part by DESY, that is devoted to studying advanced techniques of calorimetry for the ILC. The installation of the CALICE apparatus in the test beam in April, 2008 was substantial, involving veto detectors, drift chambers, electromagnetic calorimetry, hadronic calorimetry, and muon identification. A photo of the final setup is shown in Figure 15. The finely granulated hadronic calorimeter was useful in identifying beam species. The figure also shows the measurements of relative flux of particle species that CALICE determined by analyzing their data.
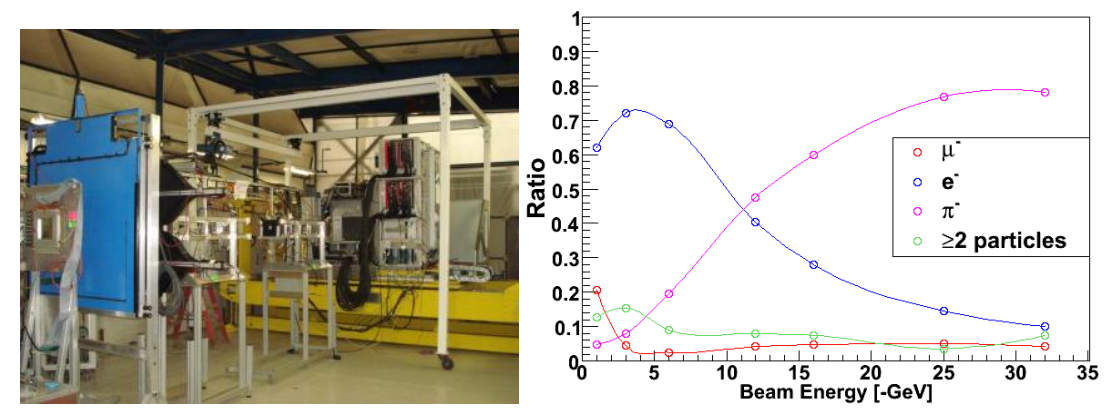

Figure 15. Left: the CALICE experiment setup in the MTest Facility. Right: composition as determined by CALICE.

\section{Developing a new tertiary low-energy beamline in the MTest facility}

The MINER $v$ A experiment (E-938) proposed installing an entirely new tertiary beamline in the user facility to deliver $300 \mathrm{MeV} / \mathrm{c}$ pions onto their test apparatus (T-977). The installation of this beamline was begun in FY 2008 and was completed in the fall of 2009. A schematic drawing and photograph of the new beamline is shown in Figure 16. After completion of the MINERvA tests in spring 2010, this beamline will be available for other users. The target and collimator can be rolled quickly aside so that the facility can also operate as before. 


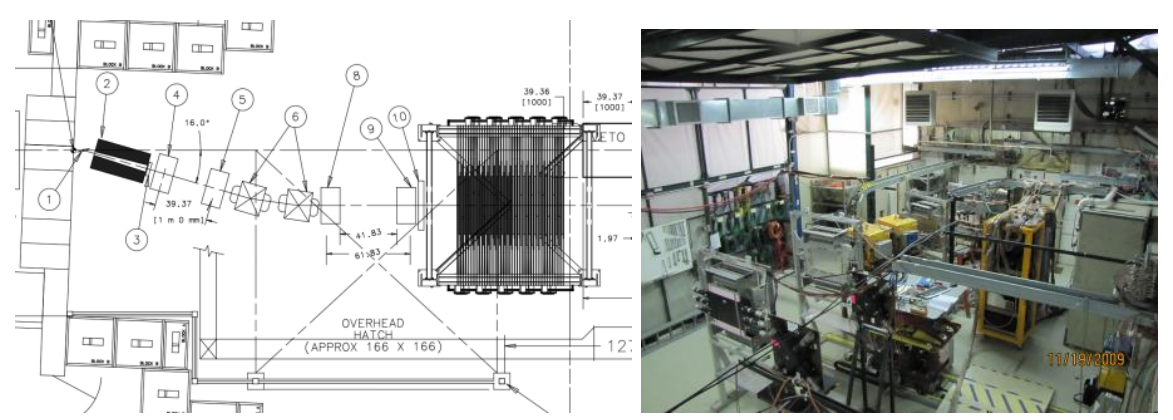

Figure 16. Schematic drawing and photo of the MINERvA tertiary beamline installation. The MINERvA test apparatus is shown in the drawing, but was not yet installed at the end of FY 2009. 